\title{
Confiabilidade intra e interavaliador por fotogrametria para avaliação do ângulo poplíteo
}

\author{
Intra- and inter-examiner reliability for popliteal angle assessment \\ by photogrammetry
}

\author{
Camila Lima Ribeiro'; Mateus Neiva Martins'; Lívia Lúcio de Mattos Amaro'; Sávia Alves Pinto1; \\ Alexandre Wesley de Carvalho Barbosa²; Renato Aparecido de Souza ${ }^{3}$; Murilo Xavier Oliveira \\ ${ }^{1}$ Graduação em Fisioterapia - UFVJM. Diamantina, MG - Brasil. \\ ${ }^{2}$ Fisioterapeuta, Doutorando em Ciências Biomédicas pelo Instituto Universitário Italiano de Rosário; Docente do Curso de \\ Fisioterapia - UFVJM. Diamantina, MG - Brasil. \\ ${ }^{3}$ Fisioterapeuta, Doutor em Engenharia Biomédica, Docente do Instituto Federal de Educação, Ciência e Tecnologia do Sul de Minas \\ Gerais. Muzambinho, MG, Brasil. \\ ${ }^{4}$ Fisioterapeuta, Doutorando em Engenharia Biomédica, Docente do Curso de Fisioterapia - UFVJM. Diamantina, MG - Brasil.
}

Endereco para correspondência

Murilo Xavier Oliveira

Rodovia MGT 367, km 583, n 5000, Alto da Jacuba

39100-000 - Diamantina - MG [Brasil]

muriloxavier@gmail.com

\section{Resumo}

Introdução: Ângulos utilizados pela fotogrametria carecem de confiabilidade para uso rotineiro. Objetivo: Avaliar a confiabilidade inter e intra-avaliador da fotogrametria pelo software SAPO do ângulo poplíteo. Métodos: Participaram 20 voluntários. Realizaram-se três medidas goniométricas e fotografias tomadas do goniômetro e ângulo poplíteo (M1). Três avaliadores analisaram as fotografias pelo SAPO. As mensurações foram repetidas 48 h após M1 (M2), e os dados analisados pelo teste " $t$ " pareado e independente, pelo coeficiente Pearson $(r)$, pelo coeficiente de variação (CV) (reprodutibilidade relativa e absoluta, respectivamente) e a consistência pelo coeficiente de correlação intraclasse (ICC), com p<0,05. Resultados: $\mathrm{O}$ teste " $\mathrm{t}$ " não detectou diferença entre os resultados. $\mathrm{O} r$ variou de 0,9031 a 0,9983 , com $\mathrm{p}<0,0001$. O ICC goniométrico e fotogramétrico foi muito bom e excelente $(\mathrm{p}<0,0001)$. O CV apresentou valor interexaminadores de $0,21 \%$, em M1, e 0,17\%, em M2, e intraexaminador de 1,32\%. Conclusão: A confiabilidade inter e intra-avaliadores foi estabelecida para avaliação do ângulo poplíteo.

Descritores: Amplitude de movimento articular; Fisioterapia; Goniometria articular.

\begin{abstract}
Introduction: Angles used by photogrammetry need reliability studies for routine use. Objective: To evaluate the reliability intra- and inter-examiner by photogrammetry software SAPO in popliteal angle measures. Methods: Participated 20 volunteers and three goniometric measures were performed and photographs were taken, showing the goniometer and the popliteal angle (M1). Three observers examined the photos using SAPO. The measurements were repeated $48 \mathrm{~h}$ after M1 (M2) and the data analyzed by paired and independent t-test, by Pearson coefficient $(r)$, coefficient of variation (CV) (relative and absolute reliability, respectively) and consistency by intraclass correlation coefficient (ICC), with $\mathrm{p}<0.05$. Results: The t-test did not detect differences between the results. The $r$ ranged from 0.9031 to 0.9983 , with $\mathrm{p}<0.0001$. The goniometric and photogrammetric ICC were classified as very good and excellent $(\mathrm{p}<0.0001)$. The CV presented interexaminer data of $0.21 \%$ in M1 and $0.17 \%$ in M2 and intra-examiner data of $1.32 \%$. Conclusion: Reliability inter and intra-examiners were established to evaluate popliteal angle.
\end{abstract}

Key words: Goniometry, articular; Physical therapy specialty; Range of motion, articular. 


\section{Introdução}

A medida da amplitude de movimento (ADM) é parâmetro determinante utilizado na avaliação e na evolução do tratamento fisioterapêutico, permitindo o acompanhamento de modo quantitativo da eficácia de intervenções terapêuticas durante um programa de reabilitação e/ou treinamento ${ }^{1}$. Além disso, faz parte da definição da propedêutica e do prognóstico de um indivíduo submetido à fisioterapia. Dentre os inúmeros métodos para mensuração da ADM, destaca-se a estimativa visual, radiografia, goniometria manual e, mais recentemente, a fotogrametria computadorizada $^{1,2,3}$.

A goniometria manual, descrita na literatura desde 1914, é amplamente usada, tanto na prática clínica quanto em pesquisas científicas, com a finalidade de medir a ADM de diversas articulações ${ }^{4,5}$. Vários experimentos examinaram o grau de confiabilidade das medidas goniométricas utilizando diferentes procedimentos de medida e demonstraram que a ADM do joelho, medida com goniômetro universal, obteve um nível de confiabilidade de bom a excelente ${ }^{4,5}$. Dentre as vantagens dessa metodologia, pode-se citar o baixo custo do instrumento e a fácil mensuração que depende quase que exclusivamente da experiência prévia do avaliador ${ }^{6}$.

Outra técnica de análise que nos últimos anos tem sido amplamente utilizada na clínica é a fotogrametria computadorizada. Definida como arte, ciência e tecnologia de obtenção de informação confiável sobre objetos físicos e o ambiente por meio de processos de gravação, medição e interpretação de imagens fotográfi$\mathrm{cas}^{7}$. A fotogrametria computadorizada possui vantagens adicionais à goniometria manual, além de permitir as comparações de fases e avaliação da eficácia de um tratamento, esse sistema facilita o processo de arquivamento, com a economia de espaço e também de tempo, no acesso aos registros arquivados, sendo facilmente acessada por computador e editada em softwares de análise de imagens ${ }^{8}$.
O software para avaliação postural (SAPO) é um programa de uso relativamente simples e gratuito, de livre acesso na internet, que fornece, além das medidas lineares, valores angulares. Fundamenta-se na digitalização de pontos espacialmente definidos que possibilita funções diversas, tais como a calibração da imagem, utilização de zoom, marcação livre de pontos, medição de distâncias e de ângulos corporais," 10 . Nesse sentido, o SAPO pode ser utilizado por um profissional habilitado como uma ferramenta auxiliar na avaliação da ADM por meio da fotogrametria computadorizada ${ }^{10}$.

Apesar da grande difusão de pesquisas utilizando a fotogrametria computadorizada para avaliar alterações posturais, são escassos estudos em que seja usada essa técnica como uma possível ferramenta para análise da ADM. Dessa forma, sua precisão ainda é questionada e sua utilização deve ser adequadamente planejada de modo a prevenir erros ${ }^{11,12}$. Portanto, o estabelecimento de critérios e testes de confiabilidade das medidas torna-se necessário para sustentar seu uso na mensuração da ADM, além disso, poucos estudos avaliaram a confiabilidade dessa ferramenta, como um novo método ou como complementar aos modelos de avaliação articulares tradicionalmente descritos.

Diante do exposto, o objetivo deste trabalho foi avaliar a confiabilidade inter e intra-avaliador da fotogrametria computadorizada por meio do software SAPO, nas medidas do ângulo poplíteo.

\section{Materiais e métodos}

\section{Considerações éticas}

Trata-se de um estudo observacional transversal, aprovado pelo Comitê de Ética em Pesquisa da Universidade Federal dos Vales do Jequitinhonha e Mucuri (UFVJM), sob protocolo $n^{\circ} 172 / 10$. Para a realização do trabalho, foi solicitado que os voluntários assinassem um Termo de Consentimento Livre e Esclarecido, conforme Resolução 196/96 do Conselho Nacional de Saúde. 


\section{Amostra}

Participaram deste estudo 20 voluntários, estudantes universitários, sendo 14 do sexo feminino; e 6, do masculino (massa corporal: 62,6 $6 \pm 12,3$ kg; estatura: 1,70 $\pm 0,10 \mathrm{~m}$; IMC: $22,50 \pm 3,36 \mathrm{~kg} / \mathrm{m}^{2}$ ), que não apresentassem dor muscular ou articular de membros inferiores e incapacidade de relaxar durante os procedimentos.

\section{Anólise da amplitude de movimento}

Os experimentos foram realizados em dois dias de coleta, com intervalo de 48 horas entre eles.

Primeiramente, posicionou-se a maca com um fio de prumo fixado para calibração da imagem digitalizada e colocou-se a máquina fotográfica digital (Kodak C813 de 8,2 megapixels) a três metros de distância sobre o centro do tripé a altura de $70 \mathrm{~cm}$ do chão. Foram marcados pontos no chão para que a maca e tripé sempre estivessem no mesmo local. Logo após, três marcadores (bolas de isopor) foram colocados no goniômetro universal da marca $\mathrm{CARCI}^{\circledR}$, (fulcro, centro da haste fixa e centro da haste móvel estendida) e a região da haste móvel, onde contém os valores angulares, foi tampada com cartolina.

O voluntário foi orientado a sortear, com utilização de envelope lacrado, qual perna seria examinada, e a posicionar-se em supino, na maca, com o membro de teste voltado para a máquina fotográfica. Foram marcados com bolas de isopor os seguintes pontos anatômicos antes da mensuração: trocânter maior e côndilo lateral do fêmur (com marcador adesivo de papel) e o maléolo lateral da fíbula, sempre pelo mesmo avaliador.

A fim de evitar movimentos compensatórios da pelve, do tronco e do membro contralateral foi utilizada atadura de crepom de $8 \mathrm{~cm}$ de espessura que envolveram e fixaram o indivíduo na maca, na altura da espinha ilíaca anterossuperior ao nível médio da coxa oposta.

$\mathrm{O}$ primeiro avaliador realizou, no sujeito, uma flexão de quadril a $90^{\circ}$ (medida monitorada por um goniômetro universal, já descrito), e a partir daí estendeu o joelho até encontrar o ponto de li- mite máximo de movimento. Logo após, o segundo avaliador posicionou o goniômetro para mensuração da ADM, sem ter consciência do valor obtido, e o terceiro registrou a imagem pela fotografia. Todo esse procedimento foi realizado três vezes consecutivas por avaliadores previamente treinados.

Para maior confiabilidade dos dados encontrados, a leitura do goniômetro foi sempre realizada e registrada por um mesmo avaliador. Além disso, para mais segurança, sempre foi outro pesquisador quem fotografou com os mesmos padrões de focalização.

As imagens foram analisadas por três pesquisadores, o segundo que posicionou o goniômetro, o terceiro que fotografou, além de um avaliador voluntário do estudo. Os três foram previamente treinados ao manuseio do software SAPO (versão 0.68), com o qual mensuraram, de forma cega, o ângulo poplíteo de três fotos adquiridas, baseando nas marcações de isopor fixadas no goniômetro sem que soubessem os valores reais encontrados por este (Figura 1). Esse procedimento foi realizado em dois momentos distintos: M1, ou seja, logo após o procedimento de fotografia; e M2, 48 horas após M1.

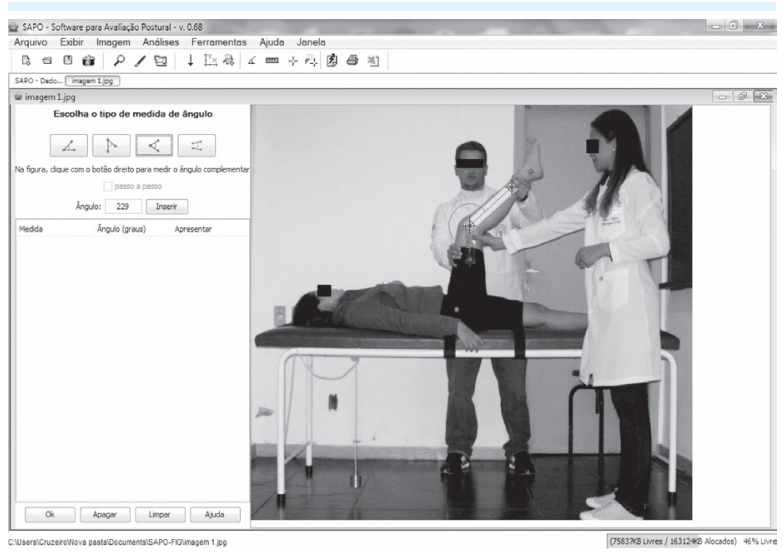

Figura 1: Posicionamento de voluntário, prumo (fixado na maca) e avaliadores para a mensuração do ângulo poplíteo

\section{Anólise estatística}

Para a análise estatística, foi utilizado o software Bioestat (versão 5.0, Belém, PA) e os valores angulares obtidos por todos os examinadores foram testados quanto à normalidade pelo teste 
de Shapiro-Wilk. Média e desvio-padrão foram utilizados como medida de tendência central e dispersão, respectivamente. Em seguida, os parâmetros obtidos em M1 e M2 por goniometria (intraexaminador) foram comparados pelo teste " $t$ " para amostras pareadas. $O$ teste " $t$ " independente foi utilizado para comparar diferenças interexaminadores tanto em M1 como em M2.

A reprodutibilidade relativa, que define o grau em que cada indivíduo mantém sua posição em uma amostra de medidas repetidas ${ }^{13}$, foi medida pelo coeficiente de correlação de Pearson.

A reprodutibilidade interavaliador, ou seja, o fato de um ângulo dar o mesmo resultado entre observadores diferentes, foi avaliada pelo coeficiente de correlação intraclasse (ICC), assim como a reprodutibilidade intra-avaliador, sendo ranqueados conforme Wahlund et al. ${ }^{14}(0,0$ a 0,70 $=$ não aceitáveis; 0,71 a $0,79=$ aceitáveis; 0,80 a 0,89 $=$ muito bons; $>0,90=$ excelentes). A reprodutibilidade absoluta define o grau em que as medidas repetidas variam por indivíduo ${ }^{13}$ e foi verificada pelo coeficiente de variação $(\mathrm{CV})$, obtido pela razão entre o desvio-padrão e a média multiplicada por 100 (CV = desvio-padrão / média $\times 100)$. Esse procedimento foi adotado para cada caso para que, então, fosse calculado o CV de todo o grupo para cada parâmetro. Em todas as situações o nível de significância adotado foi o de $\mathrm{p}<0,05$.

\section{Resultados}

A Tabela 1 demonstra a média e o desviopadrão do ângulo poplíteo por fotogrametria intra e interexaminadores. A avaliação da confiabilidade desenvolvida pelo mesmo avaliador em dias diferentes mostrou, na Tabela 2, a comparação pareada, pelo teste " $t$ ", entre as duas mensurações e nenhuma diferença significativa foi detectada.

O teste " $\mathrm{t}$ " independente, utilizado nas comparações interexaminador, também não detectou diferença entre os resultados angulares obtidos (Tabelas 3 a 8). A confiabilidade relativa, dada como nível no qual cada sujeito mantém sua posição na amostra, foi confirmada tanto para ava-
Tabela l: Média e desvio-padrão (DP) do ôngulo poplíteo intra e interexaminador

\begin{tabular}{ccccc}
\hline & $\begin{array}{c}\text { Intraexaminador } \\
\text { (Goniometria) }\end{array}$ & \multicolumn{3}{c}{ Interexaminador (Fotogrametria) } \\
\hline & Avaliador 1 & Avaliador 1 & Avaliador 2 & Avaliador 3 \\
& Média/DP & Média/DP & Média/DP & Média/DP \\
M1 & $147,43 \pm 6,54$ & $147,67 \pm 7,95$ & $147,56 \pm 7,75$ & $147,75 \pm 7,86$ \\
M2 & $146,36 \pm 5,82$ & $147,26 \pm 7,05$ & $147,19 \pm 7,03$ & $147,14 \pm 7,07$ \\
\hline
\end{tabular}

Tabela 2: Confiabilidade intraexaminador para goniometria

\begin{tabular}{|c|c|c|c|c|c|}
\hline & $\begin{array}{c}\text { Teste "t" } \\
\text { pareado } \\
\text { (valor de p) }\end{array}$ & $r$ & ICC & $\begin{array}{l}\text { Limite } \\
\text { inferior }\end{array}$ & $\begin{array}{l}\text { Limite } \\
\text { superior }\end{array}$ \\
\hline M1xM2 & 0,1061 & $0,9031^{*}$ & $0,8851^{*}$ & 0,7275 & 0,954 \\
\hline
\end{tabular}

liação intra quanto para interexaminadores, como visto no resultado altamente significativo do coeficiente de correlação de Pearson $(\mathrm{p}<0,0001$, Tabela 2 a 8). A consistência entre as duas medidas foi classificada como excelente para o ângulo medido com goniômetro ou pela fotogrametria, apresentando um ICC com nível de significância bastante alto em todas as situações apresentadas $(\mathrm{p}<0,0001)$.

A reprodutibilidade absoluta tem sido considerada aceitável quando o coeficiente de variação (CV) se situa abaixo de $10 \%$ e, apesar de este valor ainda ser mérito de conjectura, também foi alvo de análise neste estudo. O CV intraexaminador apresentou valor de $1,32 \%$; e o mesmo dado interexaminadores, valores de $0,21 \%$, em M1, e $0,17 \%$, em M2. Os gráficos nas Figuras 2 a 4 demonstram a dispersão dos valores do CV individuais, demonstrando a proximidade dos obtidos nas mensurações realizadas voluntário a voluntário.

\section{Discussão}

A confiabilidade está ligada à precisão do procedimento; é consistente, estável, com mínimo erro sistemático ou aleatório. Algumas das origens do erro relacionam-se à variação própria dos observadores, das ferramentas de medida ou 
Tabela 3: Confiabilidade interexaminadores l e 2 para fotogrametria pelo SAPO na avaliação inicial (Ml)

\begin{tabular}{cccccc}
\hline & $\begin{array}{c}\text { Teste "t" } \\
\text { indepen- } \\
\text { dente } \\
\text { (valor de p) }\end{array}$ & r & ICC & $\begin{array}{c}\text { Limite } \\
\text { inferior }\end{array}$ & $\begin{array}{c}\text { Limite } \\
\text { superior }\end{array}$ \\
\hline $\begin{array}{c}\text { Ava1 } x \\
\text { Ava2 }\end{array}$ & 0,4923 & $0,9965^{*}$ & $0,9977^{*}$ & 0,9941 & 0,9991 \\
\hline
\end{tabular}

${ }^{*} \mathrm{p}<0,0001$.

Ava1 $=$ Avaliador 1; Ava2 $=$ Avaliador 2; $r=$ coefi ciente de correlação de Pearson; ICC = coeficiente de confiabilidade intraclasse.

Tabela 4: Confiabilidade interexaminadores l e 3 para fotogrametria pelo SAPO na avaliação inicial (Ml)

\begin{tabular}{cccccc}
\hline & $\begin{array}{c}\text { Teste "t" } \\
\text { indepen- } \\
\text { dente } \\
\text { (valor de p) }\end{array}$ & r & ICC & $\begin{array}{c}\text { Limite } \\
\text { inferior }\end{array}$ & $\begin{array}{c}\text { Limite } \\
\text { superior }\end{array}$ \\
\hline $\begin{array}{c}\text { Ava1 } x \\
\text { Ava3 }\end{array}$ & 0,4689 & $0,9956^{*}$ & $0,9975^{*}$ & 0,9935 & 0,999 \\
\hline
\end{tabular}

${ }^{*} \mathrm{p}<0,0001$.

Ava1 $=$ Avaliador 1 ; Ava3 = Avaliador 3; $r=$ coefi ciente de correlação de Pearson; ICC = coeficiente de confiabilidade intraclasse.

Tabela 5: Confiabilidade interexaminadores 2 e 3 para fotogrametria pelo SAPO na avaliação inicial (Ml)

\begin{tabular}{cccccc}
\hline & $\begin{array}{c}\text { Teste "t" } \\
\text { indepen- } \\
\text { dente } \\
\text { (valor de p) }\end{array}$ & r & ICC & $\begin{array}{c}\text { Limite } \\
\text { inferior }\end{array}$ & $\begin{array}{c}\text { Limite } \\
\text { superior }\end{array}$ \\
\hline $\begin{array}{c}\text { Ava2 } x \\
\text { Ava3 }\end{array}$ & 0,4766 & $0,9983^{*}$ & $0,9987^{*}$ & 0,9965 & 0,9995 \\
\hline
\end{tabular}

${ }^{*} \mathrm{p}<0,0001$.

Ava2 $=$ Avaliador $2 ;$ Ava3 $=$ Avaliador 3; $r=$ coefi ciente de correlação de Pearson; ICC = coeficiente de confiabilidade intraclasse.

do que está sendo mensurado. Se a confiabilidade é baixa sua validade não pode ser determinada ${ }^{15}$.

Analisar a confiabilidade e a validade de uma ferramenta significa verificar a consistência de resultados quando a mensuração se repete em condições idênticas, e comparando-a com o padrão de referência, aceitando-se que o fisioterapeuta deve embasar sua clínica em evidências científicas. A confiabilidade das medidas demonstra sua consistência obtida por um instrumento ou por
Tabela 6: Confiabilidade interexaminadores l e 2 para fotogrametria pelo SAPO na avaliação $48 \mathrm{~h}$ após a primeira avaliação (M2)

\begin{tabular}{cccccc}
\hline & $\begin{array}{c}\text { Teste "t" } \\
\text { indepen- } \\
\text { dente } \\
\text { (valor de p) }\end{array}$ & r & ICC & $\begin{array}{c}\text { Limite } \\
\text { inferior }\end{array}$ & $\begin{array}{c}\text { Limite } \\
\text { superior }\end{array}$ \\
\hline $\begin{array}{c}\text { Ava1 } x \\
\text { Ava2 }\end{array}$ & 0,4861 & $0,998^{*}$ & $0,9978^{*}$ & 0,9943 & 0,9992 \\
\hline
\end{tabular}

${ }^{*} \mathrm{p}<0,0001$.

Ava1 $=$ Avaliador $1 ;$ Ava2 $=$ Avaliador 2; $r=$ coefi ciente de correlação de Pearson; ICC = coeficiente de confiabilidade intraclasse.

Tabela 7: Confiabilidade interexaminadores 1 e 3 para fotogrametria pelo SAPO na avaliação 48 h após a primeira avaliação (M2)

\begin{tabular}{|c|c|c|c|c|c|}
\hline & $\begin{array}{l}\text { Teste " } \mathrm{t} \text { " } \\
\text { indepen- } \\
\text { dente } \\
\text { (valor de p) }\end{array}$ & $\mathbf{r}$ & ICC & $\begin{array}{l}\text { Limite } \\
\text { inferior }\end{array}$ & $\begin{array}{l}\text { Limite } \\
\text { superior }\end{array}$ \\
\hline $\begin{array}{c}\text { Ava1 x } \\
\text { Ava3 }\end{array}$ & 0,4784 & $0,9982^{*}$ & $0,998^{*}$ & 0,9948 & 0,9992 \\
\hline \multicolumn{6}{|c|}{$\begin{array}{l}\text { * } \mathrm{p}<0,0001 . \\
\text { Ava1 = Avaliador } 1 ; \text { Ava3 = Avaliador } 3 ; r=\text { coefi- } \\
\text { ciente de correlação de Pearson; ICC = coeficiente } \\
\text { de confiabilidade intraclasse. }\end{array}$} \\
\hline
\end{tabular}

Tabela 8: Confiabilidade interexaminadores 2 e 3 para fotogrametria pelo SAPO na avaliação 48 h após a primeira avaliação (M2)

\begin{tabular}{|c|c|c|c|c|c|}
\hline & $\begin{array}{l}\text { Teste " } t \text { " } \\
\text { indepen- } \\
\text { dente } \\
\text { (valor de p) }\end{array}$ & $\mathbf{r}$ & ICC & $\begin{array}{l}\text { Limite } \\
\text { inferior }\end{array}$ & $\begin{array}{l}\text { Limite } \\
\text { superior }\end{array}$ \\
\hline $\begin{array}{c}\text { Ava2x } \\
\text { Ava3 }\end{array}$ & 0,4922 & $0,9992^{*}$ & $0,9992^{*}$ & 0,9978 & 0,9997 \\
\hline \multicolumn{6}{|c|}{$\begin{array}{l}{ }^{*} p<0,0001 \\
\text { Ava2 = Avaliador } 2 ; \text { Ava3 = Avaliador 3; } r=\text { coefi- } \\
\text { ciente de correlação de Pearson; ICC = coeficiente } \\
\text { de confiabilidade intraclasse. }\end{array}$} \\
\hline
\end{tabular}

um avaliador nas mesmas condições de avaliação. A confiabilidade intra-avaliadores é a consistência das medidas realizadas nas mesmas condições de avaliação em dois momentos diferentes. Já a confiabilidade interavaliadores vincula-se à consistência das medidas realizadas por dois examinadores diferentes.

A padronização da metodologia é parâmetro fundamental para controlar as fontes de erro, proporcionando, assim, uma medida confi- 


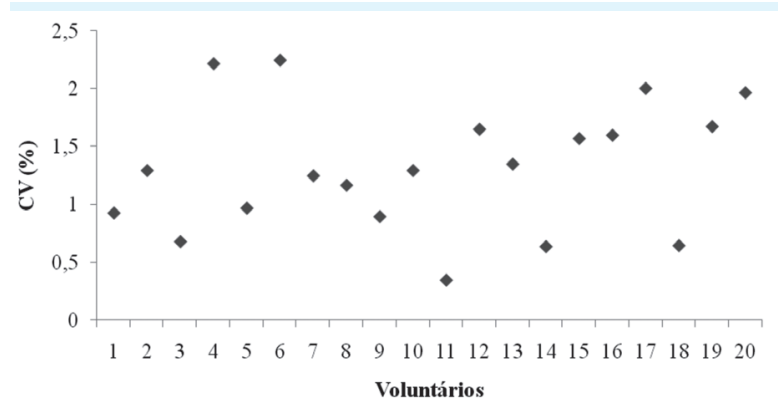

Figura 2: Coeficiente de variação individual intraexaminador entre momento 1 e 2 para mensuração de ângulo poplíteo

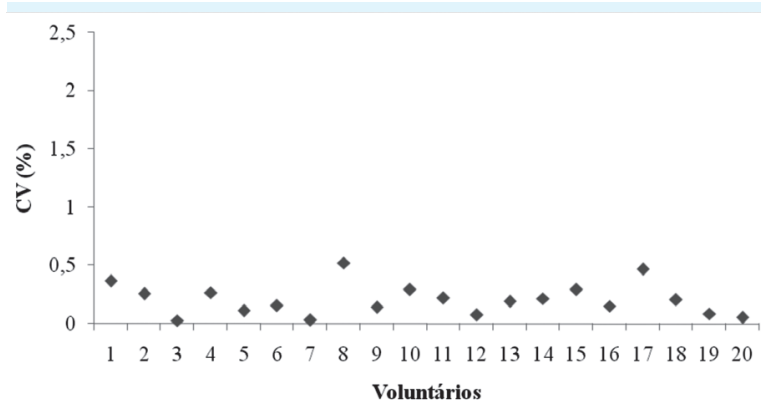

Figura 3: Coeficiente de variação individual interexaminadores no momento l para mensuração de ângulo poplíteo

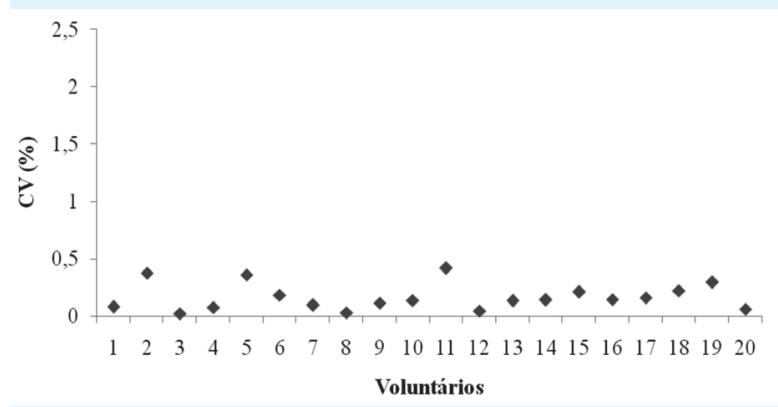

Figura 4: Coeficiente de variação individual interexaminadores no momento 2 para mensuração de ângulo poplíteo

ável ${ }^{16}$. Considerando-se ainda que a goniometria manual seja o método mais utilizado na avaliação da ADM, ela é, portanto, o instrumento referencial para que novos métodos e instrumentos, como a fotogrametria computadorizada, possam ser estudados e comparados.

Os resultados deste estudo mostraram que a análise da fotogrametria por meio do software SAPO apresentou resultados excelentes para avaliação do ângulo poplíteo, tanto intra (teste " $t$ " pareado, utilizado nas mensurações intraexa- minador, com $\mathrm{p}=0,1061$ ) quanto interavaliadores (teste " $\mathrm{t}$ " independente, com valores de $\mathrm{p}$ variando de 0,4689 a 0,4923), sem diferenças significativas entre as medidas realizadas. É razoável admitir que indivíduos altamente treinados e muito familiarizados com a mensuração proposta tendem a apresentar uma maior consistência em medidas repetidas do que os não treinados. Nesse sentido, Tedeschi et al. ${ }^{17}$ confirmam a relevância da experiência na manipulação de goniômetros, assim como de instrumentos utilizados em fotogrametria, para credibilidade dos resultados. A não significância do teste " $\mathrm{t}$ " para as medidas indica que fatores, como o efeito de aprendizagem, motivação ou inconsistência de medida angular, não influenciaram na avaliação, como também demonstrado por Sacco et al. ${ }^{8}$, em um estudo no qual o teste de diferenças entre métodos de medida (goniometria e fotogrametria) apresentou, conforme conclusões dos autores, confiabilidade moderada na medida angular do joelho no plano sagital por concordância paralela entre os métodos ( $r=0,48 ; p<0,0001)$. Observou-se no estudo supracitado, entretanto, uma baixa correlação com valor de $p$ não significativo $(r=0,06 ; p=0,6721)$ entre as medidas feitas, comparando-se a goniometria com o software SAPO. A justificativa de Sacco et $a .^{8}$ é que a escala da goniometria é numeral, enquanto a do SAPO é decimal, o que poderia conferir diferenças nos resultados que podem ser expressas por essas baixas correlações. Neste mesmo estudo, o paciente assume posição ortostática, enquanto no trabalho aqui apresentado o voluntário é colocado em decúbito dorsal, o que também pode conferir diferenças, quando comparados os resultados de ambos os estudos. Não obstante, vê-se que, novamente, a confiabilidade absoluta não foi considerada no estudo supracitado, o que remete à necessidade da análise mais aprofundada dos dados para conclusões contundentes a respeito dos resultados. A validade e a importância dessa mensuração são ressaltadas em estudos de confiabilidade prévios ${ }^{13,18}$.

Alguns autores sugerem que a fotogrametria é confiável por não apresentar diferença estatística com o goniômetro universal, resultado semelhan- 
te ao encontrado no estudo mostrado. Ribeiro et al. ${ }^{16}$ propuseram avaliação inter e intra-avaliador fotopodometria bem como a confiabilidade intraavaliador da fotopodoscopia, tendo concluído, somente pelos valores de ICC e de análise de variância one way, que a fotogrametria, nesse caso, foi um recurso de análise quantitativa confiável e de fácil aplicação, tanto na clínica quanto na pesquisa científica. Carregaro et al. ${ }^{19}$ demonstraram reprodutibilidade da fotogrametria tanto intra quanto interavaliadores para o ângulo de joelho em bipedestação, constituindo-se em uma ferramenta útil de análise dos testes de flexibilidade. Esses dados concordam com o estudo apresentado, uma vez que o ângulo poplíteo constitui-se em um teste de flexibilidade, sendo encontrada confiabilidade entre as medidas realizadas. Entretanto, apenas o ICC foi utilizado como parâmetro para as conclusões do estudo em questão, denotando a validade de análises estatísticas mais aprofundadas para ratificar a confiabilidade dessas mensurações. Assim, para confirmar a confiabilidade da fotogrametria em relação à goniometria, foi realizada a mensuração do ângulo poplíteo com o goniômetro manual, em que foi possível observar consistência, por meio do ICC $=0,8851$, muito boa intra-avaliador desse instrumento e uma excelente reprodutibilidade relativa e absoluta pelos valores do coeficiente de Pearson ( $r=0,9031)$ e CV $(1,32 \%)$, respectivamente. Os dados de ICC corroboram os estudos de Souza et $\mathrm{al}^{20}$, no qual identificaram um valor de $p$ de 0,431 para comparações por análise de variância one way; e um ICC de 0,906, classificado como excelente, para o ângulo poplíteo. Entretanto, neste estudo não foram considerados dados de confiabilidade absoluta e relativa, o que pode indicar um viés importante nas conclusões a partir dele. Neste estudo, procedeu-se a análise do coeficiente de variação, que oscilou entre 0,17 e 1,32\%, com resultados de CV individuais sempre menores que 2,5\% (Figuras 1 e 2), demonstrando a confiabilidade dessas medidas e conferindo robustez para a utilização destes parâmetros em uma perspectiva clínica e de pesquisa.

Iunes et al. ${ }^{21}$ propuseram uma avaliação quantitativa das assimetrias posturais, verifican- do confiabilidade intra e interavaliador e repetibilidade do método. A análise da confiabilidade foi efetuada a partir das medidas angulares de uma mesma foto, obtidas por um único examinador em duas ocasiões (intraexaminadores) intervaladas por um mês e de uma só medida realizada por um terceiro examinador (interexaminadores). A repetibilidade do método foi analisada pelas medidas angulares tomadas por um examinador em duas fotos diferentes do mesmo sujeito, realizadas com intervalo de uma semana, com o ICC aplicado com nível de significância de 5\%. Entre os ângulos estudados, figurava o ângulo do joelho, apresentando um ICC de 0,996, classificado como excelente para confiabilidade interexaminadores, porém com resultado intraexaminador não aceitável (ICC=0,385; $p=0,0009$ ). Segundo Boldrini et al. ${ }^{22}$ possíveis erros podem interferir nos resultados intra-avaliadores, como alteração do posicionamento do voluntário, palpação, colocação das referências anatômicas e movimento da pele sobre as proeminências ósseas demarcadas durante a mensuração da goniometria. Muitos estudos que encontram alta confiabilidade interavaliadores não consideram importante a análise da confiabilidade intra-avaliador. Contudo, os resultados encontrados neste e em estudos prévios denotam a importância em se registrar o quanto uma técnica é confiável para um mesmo avaliador em momentos distintos ${ }^{16,21,23}$.

\section{Conclusão}

A fotogrametria por meio do software SAPO apresentou confiabilidade inter e intraavaliadores para mensuração da ADM do ângulo poplíteo, sendo, portanto, uma ferramenta confiável para utilização na prática clínica.

\section{Agradecimentos}

Os autores agradecem à Fundação de Amparo à Pesquisa do Estado de Minas Gerais (FAPEMIG) pelo apoio financeiro a este projeto. 


\section{Referências}

1. Norkin CC, White DJ. Medida da AM articular: um guia para goniometria. Philadelphia: FA Davis Co; 1995.

2. Baraúna MA, Canto RST, Schulz E, Silva RAV, Silva CDC, Veras MTS. Avaliação da amplitude de movimento do ombro em mulheres mastectomizadas pela biofotogrametria computadorizada. Rev Bras Cancerol. 2004;50(1):27-31.

3. Venturini C, Ituassú NT, Teixeira LM, Deus CVO. Confiabilidade intra e inter-examinadores de dois métodos de medida da amplitude ativa de dorsiflexão do tornozelo em indivíduos saudáveis. Rev Bras Fisioter. 2006;10(4):407-11.

4. Russel TN, Bandy WD. Eccentric training and static stretching improve hamstring flexibility of high school males. J athl Training. 2004;39(3):254-8.

5. Brosseau L, Balmer S, Tousignant M, O'Sullivan JP, Goudreault C, Goudrealt M. Intra and intertester reliability and criterion validity of the parallelogram and universal goniometers for measuring maximum active knee and extension of patients with knee restrictions. Arch Phys Med Rehabil. 2001;82(3):396-02.

6. Venturini C, André A, Aguilar BP, Giacomelli B. Confiabilidade de dois métodos de avaliação da AM ativa de dorsiflexão do tornozelo em indivíduos saudáveis. Acta Fisiátr. 2006;13(1):41-5.

7. Tommaselli AMG, Silva JFC, Hasegawa JK, Galo M. Fotogrametria: aplicações à curta distância. Perfil Científico-Educacional. 1999 [acesso em 2012 ago 7]:147-59 Disponível em: http://www4.fct.unesp.br/ docentes/carto/galo/www22/pdf/1999_fct40anos.pdf

8. Sacco ICN, Alibert S, Queiroz BWC, Pripas D, Kieling I, Kimura AA. Confiabilidade da fotogrametria em relação à goniometria para avaliação postural de membros inferiores. Rev Bras Fisioter. 2007;11(5):411-7.

9. Portal do Software para Avaliação Postural [homepage na internet], São Paulo: Incubadora Virtual FAPESP. Julho de 2007 [acesso em 2009 jul 2]. Disponível em: http://sapo.incubadora.fapesp.br/

10. Ferreira EAG. Postura e controle postural: desenvolvimento e aplicação de método quantitativo de avaliação postural. [tese na internet]. São Paulo: Universidade de São Paulo, 2005 [acesso em 2012 ago 8]. Disponível em: http://xa.yimg. com/kq/groups/27654579/2104018655/name/ elizabethagferreira.pdf

11. Mitchell HL, Newton I. Medical photogrammetric measurement: overview and prospects. ISPRS J Photogramm Remote Sens. 2002; 56(5):286-94.
12. Young S. Research for medical photographers: photographic measurement. J Vis Commun Med. 2002;25(3):94-8.

13. Moreira A, Maia G, Lizana CR, Martins EA, Oliveira PR. Reprodutibilidade e concordância do teste de salto vertical com contramovimento em futebolistas de elite da categoria sub-21. Rev Educ Fís. 2008;19(3):413-21.

14. Wahlund K, List T, Dworkin SF. Temporomandibular disorders in children and adolescents: reliability of a questionnaire, clinical examination, and diagnosis. J Orofac Pain. 1998;12(1):42-51.

15. Klasser GD, Okeson JP. The clinical usefulness of surface electromyography in the diagnosis and treatment of temporomandibular disorders. J Am Dent Assoc. 2006;137:763-71.

16. Ribeiro AP, Trombini F, Iunes DH, Monte VV. Confiabilidade inter e intra-examinador da fotopodometria e intra-examinador da fotopodoscopia. Rev Bras Fisioter. 2006;10(4):435-9.

17. Tedeschi MA. Goniometria: sua prática e controvérsias. Fisioter Mov. 2003;16(2):35-40.

18. Barbosa AC, Andrade RM, Moreira A, Serrão JC, Andries Júnior O. Reprodutibilidade da curva força-tempo do estilo "Crawl" em protocolo de curta duração. Rev Bras Educ Fís Esporte. 2012;26(1):37-45.

19. Carregaro RL, Silva LCCB, Coury HJC. Comparação entre testes clínicos para avaliar a flexibilidade dos músculos posteriores da coxa. Rev Bras Fisioter. 2007;11(2):139-45.

20. Souza JA, Pasinato F, Basso D, Correa ECR, Silva AMT. Biofotogrametria confiabilidade das medidas do protocolo do software para avaliação postural (SAPO). Rev Bras Cineantropom Desempenho Hum. 2011;13(4):299-305.

21. Iunes DH, Castro FA, Salgado HS, Moura IC, Oliveira AS. Confiabilidade intra e interexaminadores e repetibilidade da avaliação postural pela fotogrametria. Rev Bras Fisioter. 2005;9(3):327-34.

22. Boldrini, CM. Avaliação da confiabilidade intra e interavaliadores e intertécnicas para três instrumentos que mensuram a extensibilidade dos músculos isquiotibiais. Fit Perf J. 2009;8(5):342-8.

23. Braz RG, Goes FPD, Carvalho GA. Confiabilidade e validade de medidas angulares por meio do software para avaliação postural. Fisioter Mov. 2008;21(3):117-26 